$\underline{\text { Preprint typeset in JHEP style. - HYPER VERSION }}$

\title{
Indirect detection of neutralino dark matter candidates in anomaly-mediated supersymmetry breaking scenarios
}

\author{
Piero Ullio \\ SISSA, via Beirut 4, 34014 Trieste, Italy \\ E-mail: ullio@sissa.it
}

\begin{abstract}
We consider a model for neutralino dark matter candidates arising in anomaly-mediated supersymmetry breaking schemes, and examine its testability through the search for exotic cosmic rays produced by neutralino pair annihilations in the dark halo of the Galaxy. We find that the model is already constrained by available antiprotons and positrons measurements and may be further tested in upcoming measurements of these cosmic ray species. We show also that the monochromatic gamma-ray flux from neutralino annihilations is enhanced in this model up to two orders of magnitude with respect to alternative scenarios. The gamma-ray flux detected by the Energetic Gamma Ray Experiment Telescope in the direction of the Galactic center exceeds significantly the theoretical expectation of standard emission models. We prove that if at least $10 \%$ of this excess is due to gamma-ray radiation with continuum energy spectrum from neutralino annihilations in the model under investigation, the associated gamma-ray line will be detected by upcoming gamma-ray experiments.
\end{abstract}

KEYWORDS: particle dark matter, supersymmetry, indirect detection. 


\section{Contents}

1. Introduction 1

2. The MSSM in the AMSB scenario 2

3. Antiproton and positron fluxes 3

4. Gamma-ray flux 5

5. Conclusion 8

\section{Introduction}

The low energy phenomenology of a supersymmetric version of the standard model depends critically on the mechanism to describe supersymmetry (SUSY) breaking, still an open question. In a recently proposed scenario, SUSY is broken dynamically in a hidden sector with dominant SUSY breaking terms induced by anomalies [1, 2]. Generic predictions of the anomaly mediated SUSY breaking (AMSB) scheme are the presence of a very heavy gravitino, with mass naturally in the $100 \mathrm{TeV}$ range or heavier, and the fact that the lightest neutralino, plausibly the lightest supersymmetric particle (LSP), can be either a Wino or a Higgsino.

One of the byproducts of SUSY is the possibility for the LSP to be a dark matter candidate. From this point of view, the case of Wino- or Higgsino-like LSPs is generally regarded as not very attractive: Their thermal relic abundance is typically rather small; it is in the range compatible with the observed dark matter density only for very massive neutralinos, as heavy as $1 \mathrm{TeV}$ for Higgsinos, see, e.g., Ref [3], and a few TeVs for Winos, see, e.g., Ref. [4]. In the AMSB scenario, however, the nowadays density of the LSP is not fixed by its thermal relic density: An additional "non-thermal" source is present due to decays into LSPs of gravitinos or moduli fields, fields that parameterize a flat direction of the theory and that dominate the energy density in the early Universe [0, 6]. Fine-tuning in this mechanism is needed for the LSP to be the main dark matter component, certainly a drawback of the AMSB scheme. On the other hand, we wish to stress here that the scenario with these LSPs accounting for the dark halo of our Galaxy has several phenomenological 
implications and will be probed by upcoming experiments for large regions in the SUSY parameter space. ${ }^{1}$

There are several complementary techniques to identify dark matter neutralinos (for comprehensive reviews, see Refs. [7, 8]). Direct detection [9] of AMSB candidates was discussed in Refs. [6, 10]. We focus here on another technique: the search for exotic cosmic rays, such as high-energy gamma-rays, antiprotons and positrons, produced by neutralino pair annihilations in the Galactic halo [11]. This method looks particularly promising for neutralino dark matter candidates in the AMSB scenario, as both Winos and Higgsinos have a very high annihilation rate into gauge bosons, giving rise to strong sources of exotic cosmic rays. Indirect detection of Wino dark matter through antiproton and positron cosmic ray measurements was mentioned in Ref. [6]; here, in a generic AMSB scenario, we derive the constraints on the model from available data and comment on the detection prospects for future experiments. Even more promising is indirect detection with the next generation of $\gamma$-ray telescopes. We compute the monochromatic gamma-ray flux [12 from dark matter neutralino annihilations, a signal with no conceivable background from known astrophysical sources, and show that it is greatly enhanced for AMSB models. We compare with present data and en-light the cases in which such flux will be detected.

The outline of the paper is as follows: In Section 2 we describe briefly the particle physics model we consider. In Section 3 we discuss implications of antiprotons and positron fluxes, while in Section 4 we consider gamma-ray fluxes. Section 5 concludes.

\section{The MSSM in the AMSB scenario}

We work in the minimal supersymmetric standard model (MSSM) as defined in Refs. [13, 7]; details on our notation are given in Ref. [3], while, for quantitative prediction, we use the DARKSUSY computer code [14]. We suppose the LSP is the lightest neutralino, defined as:

$$
\tilde{\chi}_{1}^{0}=N_{11} \tilde{B}+N_{12} \tilde{W}^{3}+N_{13} \tilde{H}_{1}^{0}+N_{14} \tilde{H}_{2}^{0}
$$

The coefficients $N_{1 j}$, and hence the gaugino fraction $Z_{g}=\left|N_{11}\right|^{2}+\left|N_{12}\right|^{2}$, are obtained by diagonalizing the neutralino mass matrix; they are mainly a function of the bino and the wino mass parameters $M_{1}$ and $M_{2}$, and of the Higgsino parameter $\mu$. If $|\mu|$ is much smaller than $\left|M_{1}\right|$ and $\left|M_{2}\right|$, the lightest neutralino is Higgsino-like and $Z_{g}$ is close to zero. In the AMSB scenario the gaugino mass parameters are predicted in terms of the gravitino mass and their ratio is set by the corresponding gauge coupling constants and $\beta$-functions coefficients of the gauge coupling constants. At the electroweak scale this relation becomes $M_{1} \simeq 3 M_{2}$, an assumption we keep

\footnotetext{
${ }^{1}$ The model has several more virtues; e.g., it solves the so-called "cosmological moduli problem", see Ref. [6].
} 
throughout the paper. If $\left|M_{2}\right|$ is much smaller than $|\mu|, Z_{g}$ is close to 1 and the lightest neutralino is Wino-like. We recall that, on the contrary, in alternative SUSY breaking scenarios, the GUT relation $M_{1} \simeq 0.5 M_{2}$ is usually assumed and a gauginolike lightest neutralino is forced to be Bino-like.

For our purpose, it is not necessary to specify the whole mass spectrum according to a specify AMSB model. The cosmic ray yield per LPS pair annihilation is fixed by the annihilation cross section and its branching ratios. For both Winos and Higgsinos, the annihilation rate is fairly large (total rate $\sigma v \sim 10^{-24} \mathrm{~cm}^{3} \mathrm{~s}^{-1}$ ), and dominated by gauge boson final states. Our predictions are then insensitive to most parameters in the sfermion and Higgs sectors, unless the annihilation cross section is enhanced further in some special configuration. We make a sample parameter choice which removes this possibility: We assume that all sfermions are degenerate in mass, with masses 10 times the lightest neutralino mass and with no-mixing between rightand left-handed components. In the Higgs sector, we fix the pseudoscalar Higgs $A$ to be heavy, $m_{A}=500 \mathrm{GeV}$ and keep $\tan \beta$ as a free parameter. Finally, to specify a model, rather than assigning $M_{2}$ and $\mu$, we fix the lightest neutralino mass and gaugino fraction $M_{\chi}$ and $Z_{g}$, and the sign of $\mu$.

\section{Antiproton and positron fluxes}

Although antimatter seems to be scarce in the observed Universe, a small flux of cosmic ray antiprotons and positrons is expected from the interaction of primary cosmic rays with the interstellar medium (see, e.g., Ref. [15]). The measured $\bar{p}$ flux (116, 17] and references therein) is compatible with the standard prediction for this secondary component, while strong exotic $\bar{p}$ sources can be ruled out. An analogous conclusion follows from $e^{+}$data (《18 and references therein).

The neutralino-induced source of exotic cosmic rays is not fully specified once the particle physics model is chosen. The source function is proportional to the square of the neutralino density locally in space, a distribution which is only loosely known. In case of charged cosmic rays, the induced flux at earth is dominated by nearby sources; hence, for a given AMSB model, constraints on the local distribution of neutralinos can be derived.

We estimate the neutralino induced $\bar{p}$ flux according to the analysis in Ref. [19], in which an accurate simulation of the antiproton yield per annihilation is performed and the propagation of antiprotons in the Galaxy is treated in a diffusive two-zone model. We suppose first that neutralinos are smoothly distributed in a spherical dark halo and compare with the data collected by the BESS [16] and CAPRICE [17] experiments in their latest flight to derive the upper bound $\rho$ on the local neutralino density. In Fig. \& we plot, with a solid line, a few isolevel curves for $\rho$ in the plane gaugino over higgsino fraction $Z_{g} /\left(1-Z_{g}\right)$ versus neutralino mass $M_{\chi}(\mu>0$ and $\tan \beta=3$ are assumed; alternative choices give hardly distinguishable curves). 


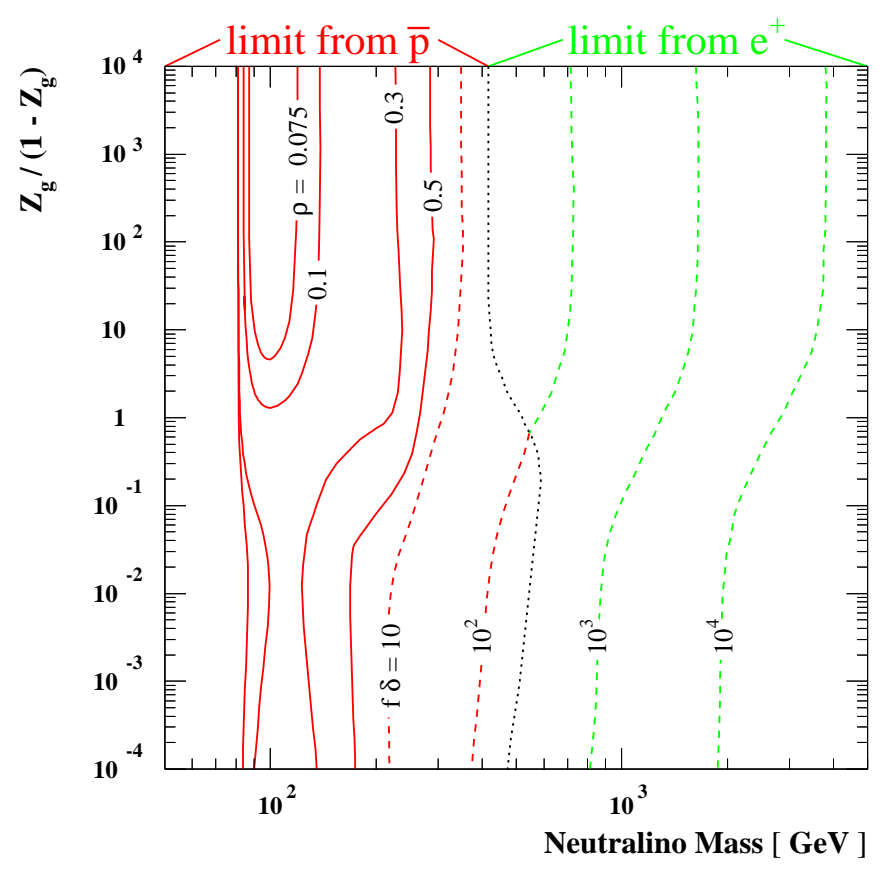

Figure 1: Isolevel curves for the maximal local halo density $\rho$ (solid lines) and maximal clumpiness parameter $f \delta$ (dashed lines), for neutralino dark matter candidates in the AMSB scenario, and in the plane gaugino over higgsino fraction versus neutralino mass. Limits for lower neutralino masses (on the left-hand side with respect to the dotted line) are derived from antiproton cosmic ray data, while those for larger neutralino masses follow from positron cosmic ray data.

The displayed limits are at 90\% C.L. and are derived with a $\chi^{2}$ method under a few conservative assumptions. We compute the neutralino-induced $\bar{p}$ flux in all the energy bins where data are available, but include in the statistical analysis only those bins in which the expected flux exceeds the measured flux; in such bins the secondary contribution is supposed to be subdominant and is neglected. The values of $\rho$ displayed are derived assuming the dark halo is described by an isothermal sphere with a 5 kpc core radius; a density profile singular towards the Galactic center would give more stringent limits [19. Finally, to model the diffusion of antiprotons in the Galaxy, we choose a standard set of the parameters in the propagation model compatible with measurements of other cosmic ray species (default choice in [19]); extreme values for these parameters may enhance (as well as suppress) the flux up to about $50 \%$. If one of these three assumption is changed, inducing a scaling of the maximal allowed $\bar{p}$ flux by roughly a factor $F$, the values assigned to the isolevel curves should be scaled by $\sqrt{F}$.

Fig. 1 shows that, for a given neutralino mass, Wino-like neutralinos (at the top 
in the figure) have more stringent limits than Higgsino-like (at the bottom) or mixed neutralinos. The lowest upper bound on the local density is about $0.06 \mathrm{GeV} \mathrm{cm}^{-3}$ for a pure Wino with mass about $100 \mathrm{GeV}$. There is a large region, with $M_{\chi}$ between 80 and $200 \mathrm{GeV}$, in which $\rho$ is lower than the value for the local dark matter density inferred from dynamical measurements in the Galaxy, about $0.3-0.5 \mathrm{GeV} \mathrm{cm}^{-3}$ if the dark halo is assumed to be spherical [20]; an additional dark matter component is then needed.

N-body simulations of hierarchical clustering [21] suggest that dark matter may form substructures, or "clumps", rather than being smoothly distributed in the halo (the picture we implemented so far). Larger local neutralino densities would enhance the sources of exotic cosmic rays [22]. Suppose that a fraction $f$ of the dark matter forms clumps with a some typical overdensity $\delta$, defined as:

$$
\delta=\frac{1}{0.3 \mathrm{GeV} \mathrm{cm}^{-3}} \frac{\int d^{3} r_{c l}\left(\rho_{c l}\left(\overrightarrow{r_{c l}}\right)\right)^{2}}{\int d^{3} r_{c l} \rho_{c l}\left(\overrightarrow{r_{c l}}\right)},
$$

where the integration is over the extent of the clump and $\rho_{c l}$ is the neutralino density profile in the clump (see [23] for further details). In this case, the induced cosmic-ray fluxes scale with the product $f \delta$ : comparing with the data, we have derived maximal values for this quantity. Isolevel curves for the maximal clumpiness parameter $f \delta$ are shown in Fig. 11 as dashed lines. For heavier and heavier neutralinos the limit inferred from the $\bar{p}$ flux becomes less and less stringent. We find that, for $M_{\chi}$ between 400 and $600 \mathrm{GeV}$, on the right hand side with respect to the dotted curve plotted in $Z_{g} /\left(1-Z_{g}\right)-M_{\chi}$ plane, the analogous limit from $e^{+}$cosmic ray measurements is more restrictive. This limit is derived computing the $e^{+}$flux according to the analysis in Ref. [24 and comparing it with data from the HEAT experiment [18], with the same approach as for the $\bar{p}$ flux.

In the next years, space-based experiments [25, 26] are going to measure the $\bar{p}$ and $e^{+}$fluxes with better statistics and much wider energy coverage. It will then be possible to set even more stringent limits; furthermore, data at high energies may give evidence for the presence of this exotic component. As mentioned, AMSB models annihilate mainly into gauge bosons and these channels automatically produce distinctive features in the high energy spectra if the neutralino-induced fluxes are at the level of the secondary components: a bump in the $e^{+}$spectrum is expected at an energy roughly equal to $M_{\chi} / 2$ [27, 24], a break in the $\bar{p}$ spectrum may appear at few tens of $\mathrm{GeV}$ 28]. In addition, a low energy exotic component in the $\bar{p}$ flux might be identified [11].

\section{Gamma-ray flux}

Gamma-rays with continuum energy spectrum are generated by the decay of $\pi^{0}$ mesons produced in jets from neutralino annihilations. It is unfortunately a rather 
featureless flux, difficult to discriminate from other plausible sources. When compared to the measured high-latitude gamma-ray background, it gives limits on the neutralino distribution in the AMSB scenario which are less restrictive than the correspondent bounds we derived from $\bar{p}$ and $e^{+}$measurements. A much better signature than the continuum contribution is given by the monochromatic $\gamma$-ray lines which arise from the 1-loop-induced neutralino annihilation into the $2 \gamma$ and $Z \gamma$ final states (the energy of the $\gamma$-rays in the final state is equal,respectively, to $M_{\chi}$ and $\left.M_{\chi}-M_{Z}^{2} / 4 M_{\chi}\right)$. Only recently, the amplitude of these processes was computed at full one loop level and for a generic MSSM [29, 30]. Predictions for these two cross sections, in the AMSB scenario and as a function of neutralino mass, are shown in Fig. 2. We have chosen a few values of $Z_{g}$ (one for each shaded area as indicated in the figure) and varied $\tan \beta$ between 1 and 60 (spread in each shaded area). The main contribution to the cross section comes from diagrams with charginos and $W$ bosons in the internal loops; these are sensitive to the mass splitting between the lightest neutralino and the lightest chargino, which is slightly modified by $\tan \beta$. In all cases the cross sections are fairly large; for Wino-like neutralinos they are a couple of orders of magnitude larger than the maximal values in other SUSY breaking scenarios [31]. The case $Z_{g}=10^{-2}$ corresponds to a good accuracy to the lower limit for each of the two cross sections; this is again a peculiarity of the AMSB scenario, as, on the other hand, there is essentially no lower bound to both cross sections for Bino-like dark matter candidates. Note also that in most cases the annihilation rate into the $Z \gamma$ final state is larger than twice the annihilation rate into two photons.

The $\gamma$-ray flux in a given direction of the sky is obtained summing the contributions along the line of sight; the largest flux is expected in the direction of the Galactic center, where an enhancement in the dark matter density is usually postulated. The distribution of dark matter in the inner part of the Galaxy is still a controversial issue. N-body simulations find singular profiles, scaling as $1 / r$ [32] or $1 / r^{1.5} 33$ as the galactocentric distance $r \rightarrow 0$. These profiles correspond to snapshots of the Galaxy before the baryon infall; the appearance of the massive black hole at the Galactic center and of the stellar components may have sensibly modified such pictures with further enhancements (but a depletion is possible as well) in the central dark matter density [34, 35]. There may be even the possibility that density of neutralinos in the galactic center region is substantial, while their contribution to the local dark matter density is subdominant. We parameterize the dependence of the $\gamma$-ray flux on the neutralino distribution through the dimensionless quantity:

$$
J(\psi)=\frac{1}{8.5 \mathrm{kpc}} \int_{\text {l.o.s. }} d l(\psi) \frac{\rho^{2}(l)}{\left(0.3 \mathrm{GeV} \mathrm{cm}^{-3}\right)^{2}},
$$

where $\rho(l)$ is the neutralino density at the distance 1 from the Earth along the line of sight. Then, we introduce $j=\langle J(\psi=0)\rangle_{\Delta \Omega=10^{-3} \mathrm{sr}}$, the average of $J$ over the field of view $\Delta \Omega=10^{-3}$ sr and in the Galactic center direction $\psi=0$. E.g., the 


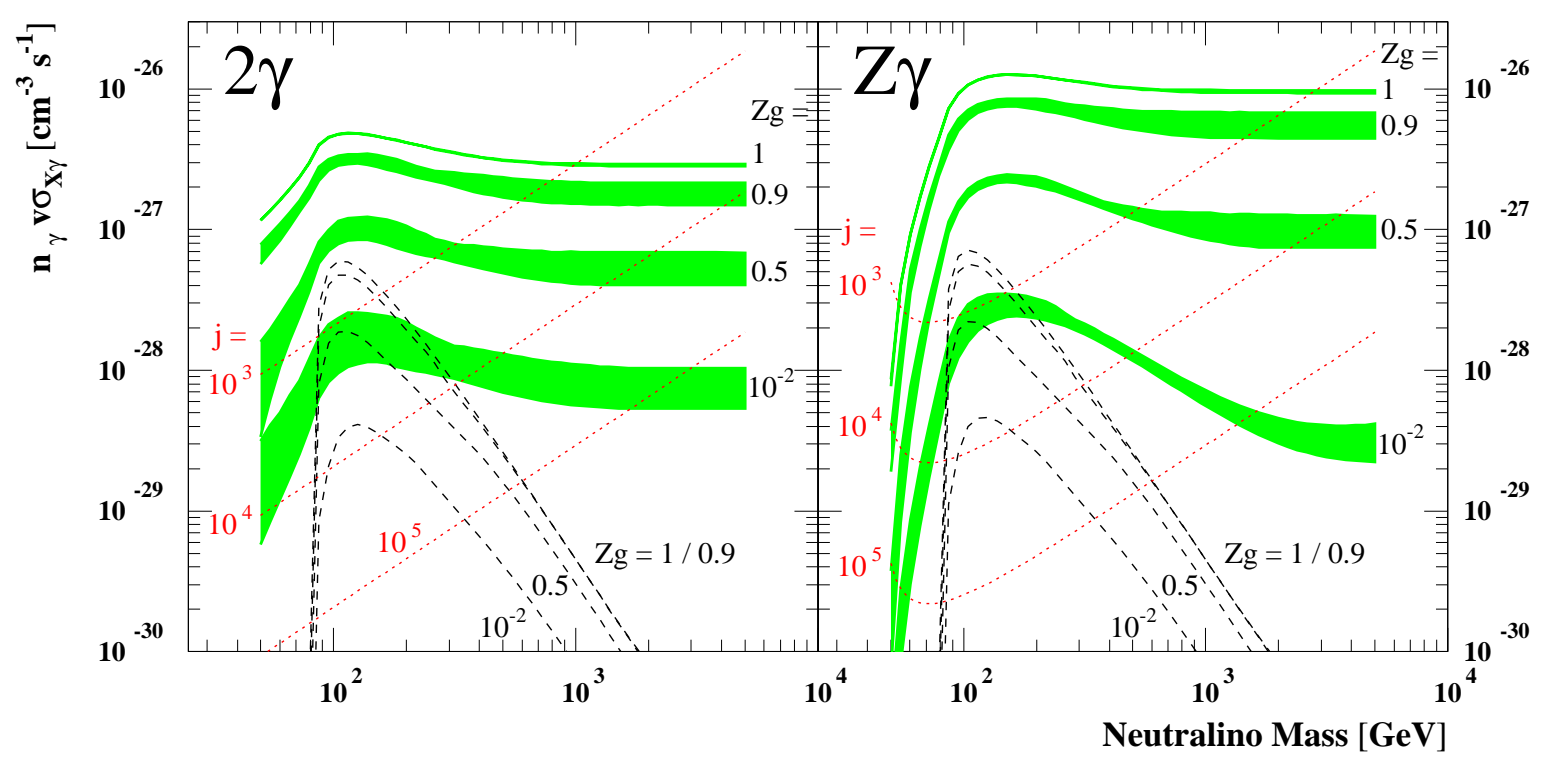

Figure 2: Annihilation rate into the two photon and the photon- $Z$ boson final states for neutralinos in the AMSB scenario versus neutralino mass; a few values of the gaugino fraction $Z_{g}$ are considered. Also shown are $5 \sigma$ sensitivity curves for a generic next generation air Cherenkov telescope [36, 37, 38] observing the Galactic center: Dotted lines correspond to a few sample dark matter distribution, including a Moore et al. profile 33 for $j=10^{5}$ and a NFW profile [32 for $j=10^{3}$. Dashed lines are obtained, for each value of $Z_{g}$, by fixing the neutralino distribution in such way that the neutralino-induced $\gamma$-ray flux with continuum energy spectrum is compatible with the excess found in EGRET data [11].

Moore et al. profile [33], scaling as $1 / r^{1.5}$ and normalized to $0.3 \mathrm{GeV} \mathrm{cm}^{-3}$ at the Sun's galactocentric distance, gives $j$ equal to about $10^{5}$; for the Navarro Frenk and White profile [32], scaling as $1 / r$ and with the same normalization, $j \sim 10^{3}$. For a given $j$, we can derive the sensitivity curve for a typical air Cherenkov gamma-ray telescope presently under development [36, 37, 38]: we consider an instrument with a $10^{9} \mathrm{~cm}^{2}$ effective area and $15 \%$ energy resolution, assume a $10^{6} \mathrm{~s}$ exposure, and use a standard estimate for the background (see [31] for details). We plot the relative $5 \sigma$ sensitivity curves for a few values of $j$ in Fig. 自 as dotted lines; sensitivity curves for the upcoming Gamma-ray Large Area Space Telescope (GLAST) [39] are comparable. As it can be seen, even for moderate values of $j$, large portion of the parameter space will be tested.

The Energetic Gamma Ray Experiment Telescope (EgRET) has mapped the diffuse Galactic gamma-ray flux up to an energy of about $20 \mathrm{GeV}$ [40]. The flux 
detected in the direction of the Galactic center exceeds significantly the theoretical expectation of standard $\gamma$-ray emission models [41]. One of the conceivable interpretation of the data is the presence of an exotic component from the annihilation of dark matter particles [41]. If we invoke the case of AMSB models, for given $M_{\chi}$ and $Z_{g}$, we can infer the maximal value of $j$ such that the neutralino-induced gamma-ray flux with continuum energy spectrum is consistent with or do not exceed the flux inferred from the data analysis. The line and continuum energy fluxes scale according to the same factor $j$; we can now consider this upper limit, say $j_{\max }\left(M_{\chi}, Z_{g}\right)$, derived from the continuum energy flux, and compute the corresponding sensitivity curves for the monochromatic fluxes (same assumptions as in the example above). These curves are shown in Fig. 2$]$ as dashed lines, one for each value of $Z_{g}$. Comparing each curve with the prediction for the line cross sections, we can formulate a rather strong statement: If at least $10 \%$ of the excess found by EGRET is due to the gamma-ray radiation with continuum energy spectrum from a neutralino dark matter candidate in a AMSB model, the associated gamma-ray lines will be detected by future gamma-ray experiments for any values of the SUSY parameters in the model. Finally, by comparing the sensitivity curves obtained for a fixed $j$ with those obtained by fixing the continuum energy flux, we find that some combination of SUSY parameters and dark matter distributions are already inconsistent with gamma-ray measurements in the Galactic center direction. Consider one sensitivity curve for a given $j$ (dotted line) and one labelled by a value of $Z_{g}$ (dashed line): values of $M_{\chi}$ between the intersection points of the two curves are excluded (unless we invoke an efficient mechanism to absorb GeV gamma-rays in the Galactic center region).

\section{Conclusion}

We considered models for neutralino dark matter candidates arising in anomaly mediated supersymmetry breaking scenarios, and discussed their testability through the search of exotic cosmic rays produced by neutralino annihilations in Galactic halo. We have shown that available cosmic ray data already place significant constraints, while even tighter limits are expected with data from upcoming experiments. At the same time, new data, both on the antiproton and positron cosmic ray flux as well as from gamma-ray surveys, may lead to the identification of these dark matter candidates. We proposed indirect detection through the search of a monochromatic $\gamma$-ray flux in the direction of the Galactic center. We showed that such flux may be observable if there is a moderate enhancement of the dark matter density in the Galactic center region, and will be detected if the excess in the gamma-ray radiation from the Galactic center found in EGRET data is caused by self annihilations of dark matter candidates in this class of models. 


\section{Acknowledgments}

The author would like to thank Lars Bergström and Joakim Edsjö for discussions.

This work was supported by the RTN project under grant HPRN-CT-2000-00152.

\section{References}

[1] L. Randall and R. Sundrum, Nucl. Phys. B 557 (1999) 79 hep-th/9810155.

[2] G.F. Giudice, M.A. Luty, H. Murayama and R. Rattazzi, J. High Energy Phys. 9812 (1998) 027 hep-ph/9810442.

[3] J. Edsjö and P. Gondolo, Phys. Rev. D 56 (1997) 1879 hep-ph/9704361.

[4] S. Mizuta, D. Ng and M. Yamaguchi, Phys. Lett. B 300 (1993) 96 hep-ph/9210241.

[5] T. Gherghetta, G.F. Giudice and J.D. Wells, \begin{tabular}{|llllll} 
Nucl. Phys. B & $\mathbf{5 5 9}$ & (1999) & 27 \\
\hline
\end{tabular} hep-ph/9904378.

[6] T. Moroi and L. Randall, Nucl. Phys. B 570 (2000) 455 hep-ph/9906527.

[7] G. Jungman, M. Kamionkowski, and K. Griest, Phys. Rep. 267 (1996) 195 hep-ph/9506380.

[8] L. Bergström, Rept. Prog. Phys. 63, 793 (2000) hep-ph/0002126].

[9] M. W. Goodman and E. Witten, Phys. Rev. D 31 (1986) 3059; I. Wasserman, Phys. Rev. D 33 (1986) 2071; A.K. Drukier, K. Freese and D.N. Spergel, Phys. Rev. D 33 (1986) 3495.

[10] B. Murakami and J.D. Wells, hep-ph/0011082].

[11] J. Silk and M. Srednicki, Phys. Rev. Lett. 53 (1984) 624; F.W. Stecker, S. Rudaz and T.F. Walsh, Phys. Rev. Lett. 55 (1985) 2622.

[12] M. Srednicki, S. Theisen and J. Silk, Phys. Rev. Lett. 56 (1986) 263; L. Bergström and H. Snellman, Phys. Rev. D 37 (1988) 3737.

[13] H.E. Haber and G.L. Kane, Phys. Rep. 117 (1985) 75.

[14] P. Gondolo, J. Edsjö, L. Bergström, P. Ullio and E.Baltz, http://www.physto.se/ẽdsjo/darksusy.

[15] T.K. Gaisser, Cosmic rays and particle physics, 1990, Cambridge University Press, Canmbridge.

[16] T. Maeno et al. (Bess Collaboration), astro-ph/0010381.

[17] M. Boezio et al. (CAPRICE Collaboration), astro-ph/0103513.

[18] S.W. Barwick et al. (HeAt Collaboration), ApJ 482 (1997) L191 astro-ph/9703192. 
[19] L. Bergström, J. Edsjö and P. Ullio, ApJ 526 (1999) 215 astro-ph/9902012.

[20] E. Gates, G. Gyuk and M. Turner, ApJ 449 (1995) L123 astro-ph/9505039.

[21] B. Moore et al., ApJ 499 (1999) L5.

[22] J. Silk and A. Stebbins, ApJ 411 (1993) 439.

[23] L. Bergström, J. Edsjö, P. Gondolo and P. Ullio Phys. Rev. D 59 (1999) 043506 astro-ph/9806072].

[24] E. Baltz and J. Edsjö, Phys. Rev. D 59 (1999) 023511 astro-ph/9808243.

[25] S. Ahlen et al. (Ams Collaboration), Nucl. Instrum. Methods A350 (1994) 351.

[26] O. Adriani et al. (PAmela Collaboration), Proc. of the 26th ICRC, Salt Lake City, 1999, OG.4.2.04.

[27] M. Kamionkowski and M.S. Turner, Phys. Rev. D 43 (1991) 1774.

[28] P. Ullio, Indirect detection of neutralino dark matter, 1999, Stockholm University Ph.D. Thesis, Stockholm.

[29] L. Bergström and P. Ullio, Nucl. Phys. B 504 (1997) 27 hep-ph/9706232.

[30] P. Ullio and L. Bergström, Phys. Rev. D 57 (1998) 1962 hep-ph/9707333.

[31] L. Bergström, P. Ullio and J. Buckley, Astropart. Phys. 9 (1998) 137 astro-ph/9712318.

[32] J.F. Navarro, C.S. Frenk and S.D.M. White, ApJ 462 (1996) 563 astro-ph/9508025.

[33] S. Ghigna et al., ApJ 544 (2000) 616 astro-ph/9910166.

[34] P. Gondolo and J Silk, Phys. Rev. Lett. 83 (1999) 1719 astro-ph/9906391.

[35] P. Ullio, H.S. Zhao and M. Kamionkowski, astro-ph/0101481.

[36] M. Martinez et al. (Magic Collaboration), Proc. of the 26th ICRC, Salt Lake City, 1999, OG.4.3.08.

[37] A. Kohnle et al. (Hess Collaboration), Proc. of the 26th ICRC, Salt Lake City, 1999, OG.4.3.13.

[38] S. Bradbury et al. (Veritas Collaboration), Proc. of the 26th ICRC, Salt Lake City, 1999, OG.4.3.28.

[39] Gamma-ray Large Area Space Telescope (GLAST), homepage http://wwwglast.stanford.edu.

[40] S.D. Hunter et al., ApJ 481 (1997) 205.

[41] H.A. Mayer- Hasselwander et al., Astron. Astrophys. 335 (1998) 161. 